Thomson Scattering Measurements of Temperature and Density in a Low-Density, Laser Driven Magnetized Plasma

S. H. Glenzer, D. B. Schaeffer, D. S. Montgomery, A. S. Bondarenko, R. P. Johnson, T. Shimada, C. G. Constantin, E. T. Everson, S. A. Letzring, S. A. Gaillard, K. A. Flippo, C. Niemann

December 23, 2011

Journal of Instrumentation 
This document was prepared as an account of work sponsored by an agency of the United States government. Neither the United States government nor Lawrence Livermore National Security, LLC, nor any of their employees makes any warranty, expressed or implied, or assumes any legal liability or responsibility for the accuracy, completeness, or usefulness of any information, apparatus, product, or process disclosed, or represents that its use would not infringe privately owned rights. Reference herein to any specific commercial product, process, or service by trade name, trademark, manufacturer, or otherwise does not necessarily constitute or imply its endorsement, recommendation, or favoring by the United States government or Lawrence Livermore National Security, LLC. The views and opinions of authors expressed herein do not necessarily state or reflect those of the United States government or Lawrence Livermore National Security, LLC, and shall not be used for advertising or product endorsement purposes. 


\title{
Thomson Scattering Measurements of Temperature and Density in a Low-Density, Laser-Driven Magnetized Plasma
}

\author{
D. B. Schaeffer ${ }^{a *}$, D. S. Montgomery ${ }^{b}$, A. S. Bondarenko ${ }^{a}$, L. A. Morton ${ }^{a \dagger}$, R. P. \\ Johnson $^{b}$, T. Shimada ${ }^{b}$, C. G. Constantin ${ }^{a}$, E. T. Everson ${ }^{a}$, S. A. Letzring ${ }^{b}$, S. A. \\ Gaillard $^{c}$, K. A. Flippo ${ }^{b}$, S. H. Glenzer ${ }^{d}$, and C. Niemann ${ }^{a}$ \\ ${ }^{a}$ Department of Physics and Astronomy, University of California - Los Angeles, \\ Los Angeles, CA 90095, U.S.A. \\ ${ }^{b}$ Los Alamos National Laboratory, \\ Los Alamos, NM 87545, U.S.A. \\ ${ }^{c}$ Forschungszentrum Dresden-Rossendorf, \\ Dresden, Germany \\ ${ }^{d}$ Lawrence Livermore National Laboratory, \\ Livermore, California 94551 U.S.A. \\ E-mail: dschaefferephysics.ucla.edu
}

\begin{abstract}
We present electron temperature and density measurements from Thomson scattering on recent collisionless shock experiments on the Trident laser at Los Alamos National Laboratory. A graphite target placed inside a static magnetic field $(\lesssim 1 \mathrm{kG})$ created by a $50 \mathrm{~cm}$-diameter Helmholtz coil was ablated by a $1053 \mathrm{~nm}$ beam, which created a low-density, magnetized plasma. A separate $527 \mathrm{~nm}$ beam was used for Thomson scattering to characterize the plasma $3 \mathrm{~cm}$ radially from the target and $0.5-8.5 \mu \mathrm{s}$ after ablation. The electron temperature was found to be relatively constant over $8 \mu \mathrm{s}$ at $11-13 \mathrm{eV}$ and, combined with Rayleigh scattering, the electron density was found to be $2 \times 10^{14}-4 \times 10^{14} \mathrm{~cm}^{-3}$ over the same timescale. Several carbon emission lines were also observed in the Thomson spectrum and were utilized to independently measure the electron temperature and density and to characterize the plasma charge state.
\end{abstract}

KEYWORDS: Plasma diagnostics - charged-particle spectroscopy, Plasma diagnostics - probes, Plasma generation (laser-produced, RF, x ray-produced).

\footnotetext{
${ }^{*}$ Corresponding author.

${ }^{\dagger}$ Now at: University of Wisconsin - Madison, Madison, WI 53715, U.S.A.
} 


\section{Contents}

$\begin{array}{lr}\text { 1. Introduction } & 1\end{array}$

$\begin{array}{lr}\text { 2. Theory } & 2\end{array}$

$\begin{array}{lr}\text { 3. Experimental Setup } & 3\end{array}$

$\begin{array}{lr}\text { 4. Results and Discussion } & 5\end{array}$

$\begin{array}{lr}\text { 5. Summary } & 8\end{array}$

\section{Introduction}

Exploding plasmas in magnetic fields are a rich and complex phenomena relevant to laboratory and astrophysical environments. Laser-ablated plasmas expanding into an ambient magnetic field are important in the study of diamagnetic cavities, anomalous magnetic diffusion, plasma instabilities, and industrial plasmas [1-4]. Previous experiments in these areas have used magnetic flux, Langmuir, and Faraday rotation probes and emission spectroscopy, but few have utilized light scattering as a diagnostic. Thomson scattering, a powerful non-invasive, non-perturbative laser diagnostic [5-7], has the advantages of being a first-principles method of obtaining plasma parameters such as electron temperature and density, as well as being both highly spatially and temporally resolved. Earlier non-magnetized works have utilized Thomson scattering on either high-density $\left(>10^{18} \mathrm{~cm}^{-3}\right)$ laser-driven plasmas [8-10] or low-density $\left(<10^{15} \mathrm{~cm}^{-3}\right)$ arc or discharge plasmas $[11,12]$, while in magnetized plasmas, Thomson scattering has been used on $\theta$-piches [13] and is a standard diagnostic on tokamaks.

We present here, to our knowledge, the first application of Thomson scattering to measurements on low-density, laser-driven magnetized plasmas. Furthermore, due to the large scale of the experiment detailed below, these measurements are the first to provide electron temperature and density information from Thomson scattering several $\mathrm{cm}$ from the target and hundreds of $\mathrm{ns}$ to $\mu \mathrm{s}$ after laser ablation, and augment the small set of existing data at these spatial and temporal scales (see, for example, [14]). We observe that the electron temperature and density remain relatively stable over several $\mu \mathrm{s}$, in contrast to the behavior seen in non-magnetized plasmas. In addition, carbon emission lines present in the Thomson spectrum were utilized to provide an independent measurement of the electron temperature and density and to characterize the plasma charge state. 


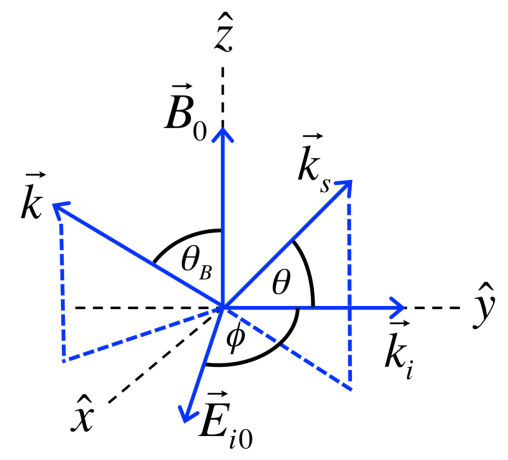

Figure 1: (Color online) Scattering coordinate system.

\section{Theory}

In optical Thomson scattering a probe source with frequency $\omega_{i}$ and wave vector $\boldsymbol{k}_{\boldsymbol{i}}$ is scattered off electron density fluctuations of wave vector $\boldsymbol{k}=\boldsymbol{k}_{\boldsymbol{s}}-\boldsymbol{k}_{\boldsymbol{i}}$ and frequency $\boldsymbol{\omega}=\omega_{s}-\omega_{i}$, where $\omega_{s}$ and $\boldsymbol{k}_{\boldsymbol{s}}$ are the frequency and wave vector of the scattered light, respectively, $\theta$ is the angle between $\boldsymbol{k}_{\boldsymbol{i}}$ and $\boldsymbol{k}_{\boldsymbol{s}}$, and for visible wavelengths $k \approx 2 k_{i} \sin (\theta / 2)$ (see Fig. 1). The scattering of visible light off density fluctuations in a non-relativistic plasma in a static magnetic field yields a scattered power $P_{s}$ into a solid angle $d \Omega$ in the frequency range $\omega_{s}$ to $\omega_{s}+d \omega_{s}$ of the form [5]

$$
P_{s} d \Omega d \omega_{s}=\frac{P_{0} r_{0}^{2} L n_{e}}{2 \pi} d \Omega d \omega_{s}\left|\hat{k}_{s} \times\left(\hat{k}_{s} \times \hat{E}_{i 0}\right)\right| S(\boldsymbol{k}, \omega)
$$

where $\left|\hat{k}_{s} \times\left(\hat{k}_{s} \times \hat{E}_{i 0}\right)\right|=1-\sin (\theta)^{2} \cos (\phi)^{2}, \phi$ is the angle between the incident polarization vector $\boldsymbol{E}_{i 0}$ and the scattering plane (see Fig. 1), $L$ is the length of the Thomson-scattering volume in the direction of the probe beam, $r_{0}^{2}=e^{2} /\left(m_{e} c^{2}\right)=2.82 \times 10^{-13} \mathrm{~cm}, n_{e}$ is the electron density, and $P_{0}$ is the incident probe power. While the full spectral density function $S(\boldsymbol{k}, \omega)$ can be found in [15], in the non-collective regime where $\alpha=\left(k \lambda_{D}\right)^{-1} \ll 1$ it can be approximated as

$$
S(\boldsymbol{k}, w)=\frac{2 \pi^{1 / 2}}{k_{\|} v_{T e}} \sum_{n=-\infty}^{+\infty} \exp \left(-k_{\perp}^{2} \rho_{e}^{2}\right) I_{n}\left(k_{\perp}^{2} \rho_{e}^{2}\right) \exp \left(-x_{n e}^{2}\right)
$$

where $x_{n e}=\left(\omega-n \Omega_{e}\right) /\left(k_{\|} v_{T e}\right), \rho_{e}=v_{T e} /\left(\sqrt{2} \Omega_{e}\right), k_{\|}=k \cos \left(\theta_{B}\right), k_{\perp}=k \sin \left(\theta_{B}\right), \theta_{B}$ is the angle between the magnetic field $\boldsymbol{B}$ and $\boldsymbol{k}$ (see Fig. 1), $\Omega_{e}$ is the electron gyrofrequency, $v_{T e}$ is the electron thermal speed, and $I_{n}$ is the modified Bessel function of the first kind. The electrons are also assumed to have a Maxwellian velocity distribution.

For any $\theta_{B}$ in the limit $B \rightarrow 0\left(k_{\perp} \rho_{e} \gg 1\right), S(\boldsymbol{k}, \omega)$ reduces to the nonmagnetic, non-collective spectral density function [16]

$$
S(\boldsymbol{k}, w)=\frac{2 \pi^{1 / 2}}{k v_{T e}} \exp \left(-x_{e}^{2}\right)
$$

where $x_{e}=\omega /\left(k v_{T e}\right)$. The primary effect of adding $B \neq 0$ is to modulate the nonmagnetic spectrum at intervals of $\Omega_{e}$. However, for small magnetic fields $\left(\Omega_{e} /\left(k v_{T e}\right) \ll \cos \left(\theta_{B}\right)\right)$, the modulation is largely washed out and can effectively be ignored [17]. 
For this experiment, $\Omega_{e} /\left(k v_{T e}\right) \approx 10^{-4} \ll \cos \left(89^{\circ}\right)$ and $\alpha \approx 0.04$, so we are justified in taking the non-collective, non-magnetic limit. In this limit, the scattered spectrum is just Gaussian in shape. The electron temperature $T_{e}$ can then be obtained from the spectrum using

$$
T_{e}=A \cdot\left(\frac{\Delta \lambda_{t h}}{\lambda_{i} \cdot \sin \left(\frac{\theta}{2}\right)}\right)^{2}
$$

where $A=\left(m_{e} \cdot c^{2}\right) /\left(8 k_{B}\right)=6.39 \times 10^{4} \mathrm{eV}, \Delta \lambda_{t h}$ is the spectral $\left(\mathrm{e}^{-1}\right)$ half-width, $\lambda_{i}$ is the probe wavelength, and $T_{e}$ is in $\mathrm{eV}$.

If the plasma has a bulk fluid speed $v_{F}$, it will appear as a frequency shift in the scattered spectrum according to $\Delta \omega^{\prime}=\omega_{o b s}-\omega_{i}=\boldsymbol{k} \cdot \boldsymbol{v}_{\boldsymbol{F}}$ or

$$
\frac{v_{F}}{c}=\left(\frac{\lambda_{i}}{\lambda_{o b s}}-1\right)\left(\frac{1}{2 \sin \left(\frac{\theta}{2}\right) \cos (\gamma)}\right)
$$

where $\lambda_{o b s}$ is the observed wavelength, and $\gamma$ is the angle between $\boldsymbol{v}_{\boldsymbol{F}}$ and $\boldsymbol{k}$. Additionally, if the electrons have a drift $v_{D}$ relative to the ions, there is a further Doppler shift of $\Delta \omega^{\prime \prime}=\hat{k} \cdot v_{D}$.

Since the scattered power is proportional to the electron density (see Eq. 2.1), with suitable calibration the electron density can be extracted from the amplitude of the Thomson-scattered spectrum. Using Rayleigh scattering off air to calibrate the optical collection system, the electron density $n_{e}$ can be obtained by

$$
n_{e}=n_{r} \cdot\left(\frac{I_{t h}}{I_{R}}\right) \cdot\left(\frac{L_{R}}{L_{t h}}\right) \cdot\left(\frac{\sigma_{R}}{\sigma_{t h}}\right)
$$

where $n_{R}$ is the air density, $I_{t h} / I_{R}$ is the ratio of the Thomson- to Rayleigh-scattered total integrated signal intensity, $L_{R} / L_{t h}$ is the ratio of Rayleigh to Thomson laser energy, and $\sigma_{R} / \sigma_{t h}$ is the ratio of the Rayleigh- to Thomson-scattering cross sections.

\section{Experimental Setup}

The experiments detailed here were carried out at the Trident laser facility [18] at Los Alamos National Laboratory. The overall layout of the experiment is shown in Fig. 2. A graphite rectangular prism target was placed at the inner edge of a $50 \mathrm{~cm}$ diameter pulsed Helmholtz coil that could create quasi-static magnetic fields up to $1 \mathrm{kG}$. The target was translated or rotated to provide a fresh surface for each shot and was ablated by two sequential laser pulses at $1053 \mathrm{~nm}$. The first ("heater") beam had a pulse width of $50 \mathrm{~ns}$ and an energy up to $100 \mathrm{~J}\left(2.5 \times 10^{11} \mathrm{~W} / \mathrm{cm}^{2}\right)$ and created an ambient, low-density, magnetized plasma. The second ("driver") beam had a pulse width of $5 \mathrm{~ns}$ and an energy up to $200 \mathrm{~J}\left(5 \times 10^{12} \mathrm{~W} / \mathrm{cm}^{2}\right)$ and created a super-Alfvénic plasma to shock the ambient plasma [19]. The delay between the pulses was variable up to $10 \mu \mathrm{s}$. An array of single-axis, non-differential $1 \mathrm{~mm}$ magnetic flux ("b-dot") probes [20] measured magnetic field compression, expulsion, and fast diffusion inside and around the diamagnetic cavity formed by the laser-plasma expansion. The line normal to the target surface along the b-dot array defines the "x"-axis, and the line vertically through the center of the Helmholtz coil along the magnetic field defines the "z"-axis (see Fig. 2c). 


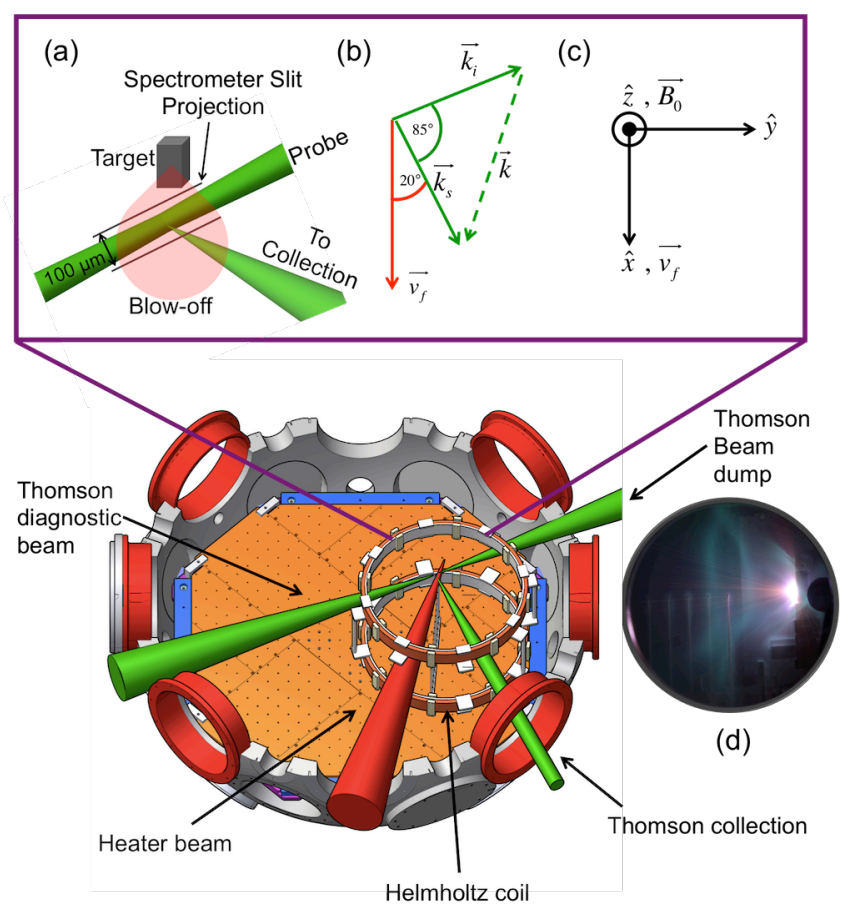

Figure 2: (Color online) Schematic layout of the experiment. (a) Cartoon of the Thomsonscattering volume. (b) Scattering wave vector geometry. (c) Coordinate system of the experiment. (d) Open-shutter photograph of target, magnetic probes, and expanding plasma.

A separate laser at $526.75 \mathrm{~nm}$ with a pulse width of $0.5 \mathrm{~ns}$ and an energy up to $60 \mathrm{~J}$ was used as a Thomson-scattering probe beam. The beam was polarized perpendicularly to the $\mathrm{x}$-y plane and was focused through a phase plate by an f/12 lens. It could be delayed arbitrarily relative to the heater or driver beams and was aimed $3 \mathrm{~cm}$ from the target surface along the $\mathrm{x}$-axis. The Thomson-scattered light was collected at an angle of $85^{\circ}$ relative to the Thomson beam axis and at an angle of $20^{\circ}$ relative to the x-axis (see Fig. 2b). The scattered light was collimated by an f/8 plano-convex lens and imaged by an f/4 achromatic lens onto an f/4 $0.25 \mathrm{~m} \mathrm{Chromex}^{\mathrm{TM}} 250$ imaging spectrometer with a 1200 grooves $/ \mathrm{mm}$ grating blazed at $500 \mathrm{~nm}$. The Thomson collection system had a magnification of 2 . The scattered light was detected by a Princeton Instruments MAX $^{\mathrm{TM}}$ intensified charge-coupled device (ICCD) gated camera with a $512 \times 512$ pixel array and an effective square pixel size of $24 \mu \mathrm{m}$. The camera was setup with a $10 \mathrm{~ns}$ exposure.

The Thomson scattering volume was defined by the probe beam waist (100 $\mu$ m radius), the projection of the spectrometer slit width onto the probe beam $(100 \mu \mathrm{m})$, and the detector CCD size in the spatial direction ( $4 \mathrm{~mm}$, see Fig. 2a). This yielded a spectral resolution of $0.6 \mathrm{~nm}$ and a spatial resolution of $7 \mu \mathrm{m}$ along the Thomson beam. A black anodized plate angled away from the collection axis served as the viewing dump, while a baffle placed in the exit port of the probe beam acted as a beam dump. A KG3 bandpass filter placed in front of the spectrometer slit blocked light above $800 \mathrm{~nm}$, including stray light from the heater and driver beams. Stray light from the probe beam was blocked by a $3 \mathrm{~mm}$ wide $(\Delta \lambda \approx 8 \mathrm{~nm}$ ) hard aperture mask placed directly in front of the detector CCD. 


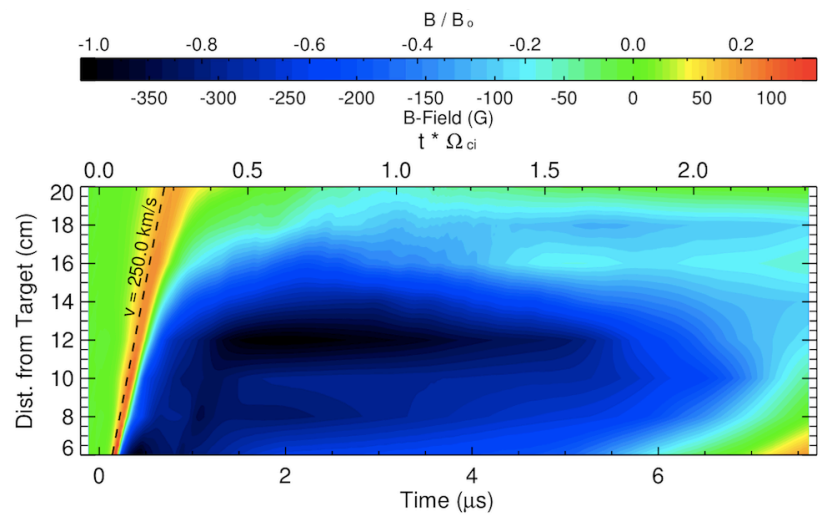

Figure 3: (Color online) Magnetic contour plot of the evolution of a diamagnetic cavity driven by the heater-ablated plasma for $B_{0}=390 \mathrm{G}$.

The Thomson collection system was aligned using a $1 \mathrm{~mm}$ diameter stainless steel pin placed inside a $1.9 \mathrm{~mm}$ diameter pyrex capillary tube to facilitate scattering. The probe beam was focused onto the tip of the pin and adjusted along the z-axis to center the pin image on the detector.

\section{Results and Discussion}

As can be seen in Fig. 3, the ablation of the target by the heater beam created a diamagnetic cavity as the blow-off plasma expanded outward [21]. The cavity was preceded by a magnetic compression pulse moving at a speed $v_{p}=250 \mathrm{~km} / \mathrm{s}$ (the initial speed of the blow-off plasma) and reached a maximum size of $\sim 20 \mathrm{~cm}$, consistent with the scaling laws for the magnetic stopping radius for a background field of $390 \mathrm{G}$ (assuming the blow-off ions lose all of their energy to the expulsion of the background field) [22]. After $\sim 7 \mu$ s, the magnetic field had completely diffused back in (though much faster than classical Bohm diffusion [1]).

To characterize the magnetized heater-driven plasma prior to the driver pulse, Thomsonscattering data was taken $3 \mathrm{~cm}$ from the target and $0.5 \mu$ s (during cavity formation) and $8.5 \mu \mathrm{s}$ (after the field diffused back in) after heater beam ablation. Since the Thomson-scattered power $P_{s}$ depends on the polarization of the probe beam (see Eq. 2.1), a second set of data was taken at 0.5 $\mu \mathrm{s}$ with the probe beam polarization rotated $90^{\circ}$ (i.e. parallel to the $\mathrm{x}$-y plane) to confirm the signal was Thomson scattering (residual scattered signal would then be stray light or Rayleigh scattering). A representative spectral profile from $0.5 \mu$ s after ablation is shown in Fig. $4 \mathrm{a}$, along with the spectral position of the mask and the corresponding spectral profile from the rotated-polarization image.

To calculate an electron temperature, background and stray light signals were subtracted, and a Voigt profile (convolution of a Gaussian and Lorentz profile to account for instrument broadening) was fit using a Levenberg-Marquardt best-fit algorithm to the non-masked signal. The error in the temperature was dominated by the accuracy of the fit (see Fig. 4b). From Eq. 2.4, the electron temperature $3 \mathrm{~cm}$ from the target and $0.5 \mu$ s after ablation was measured to be $13 \pm 2 \mathrm{eV}$. Using the electron density calculated below, this yields $\alpha=0.05 \pm 0.01$. The electron temperature at 8.5 $\mu$ s was similar at $11.5 \pm 1.5 \mathrm{eV}(\alpha=0.04 \pm 0.01)$. 

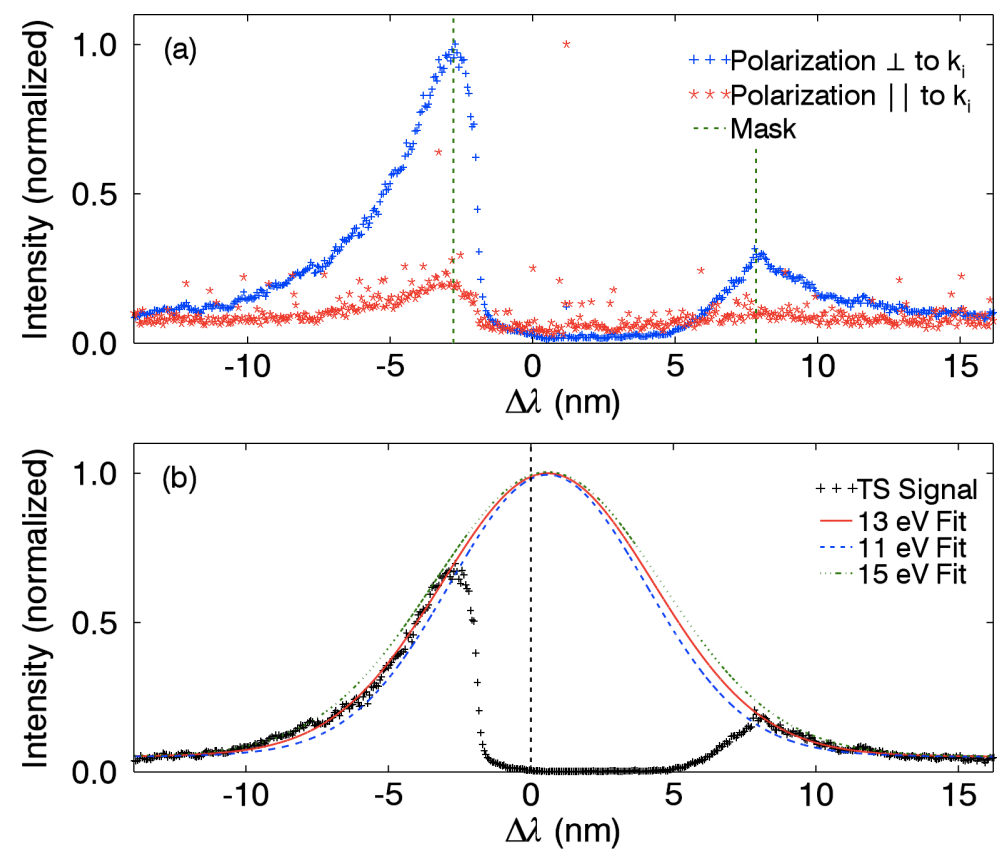

Figure 4: (Color online) (a) Representative spectral profile taken $0.5 \mu \mathrm{s}$ after ablation with probe beam polarization perpendicular (crosses) and parallel (asterisks) to the scattering plane. (b) The spectrum at $0.5 \mu$ s (crosses) fit with a Voigt profile (line). The sensitivity of the fit to electron temperature is shown by varying the electron temperature (dashed) around the best fit temperature of $13 \mathrm{eV}$.

Rayleigh scattering data was taken at chamber air fill pressures of 0.1 - 550 Torr with the Thomson probe beam at a laser energy of $50 \mathrm{~mJ}$. Since 0.1 Torr was too low for detectable Rayleigh scattering, data taken at that pressure served as a stray light and background signal fiducial while the other data were used to confirm the scattering was linear in pressure. Total integrated signal intensities were calculated by numerically integrating over the background-and-stray-light-subtracted Thomson and Rayleigh signals. Using Eq. 2.6, the electron density was calculated to be $4 \pm 2 \times 10^{14}$ $\mathrm{cm}^{-3}$ at $0.5 \mu \mathrm{s}$ and $2 \pm 1 \times 10^{14} \mathrm{~cm}^{-3}$ at $8.5 \mu \mathrm{s}$.

In addition to the Thomson-scattering signal, several carbon emission lines were also present in the spectrum. Figures $5 \mathrm{a}$ and $5 \mathrm{~b}$ show carbon ion populations $3 \mathrm{~cm}$ from the target at 0.5 and $8.5 \mu$ s after heater beam ablation, respectively. Populations of singly ionized (C II) carbon can be clearly seen at both 0.5 and $8.5 \mu \mathrm{s}$, while at $8.5 \mu \mathrm{s}$ a CIII line seen at $0.5 \mu \mathrm{s}$ is replaced by a CII line and a molecular carbon line $\left(\mathrm{C}_{2}\right.$ Swan band $\left.\Delta v=0\right)$ appears.

From the Doppler shifts of the carbon lines, the instantaneous velocities of several carbon ion species were estimated. At $0.5 \mu \mathrm{s}$, the CIII line was shifted $0.2 \mathrm{~nm}$ while the CII lines were only shifted $0.1 \mathrm{~nm}$, corresponding to ion speeds of 120 and $60 \mathrm{~km} / \mathrm{s}$, respectively. In contrast, ion velocities at $8.5 \mu$ s for $\mathrm{CII}$ and $\mathrm{C}_{2}$ were effectively zero since the doppler shifts were smaller than the calibration errors. This is consistent with the heater-driven blow-off ions coming to a stop as they lose energy to electrons that form a diamagnetic current and expel the magnetic field (see Fig. 3).

The carbon spectra were used to independently check the electron temperature and density 

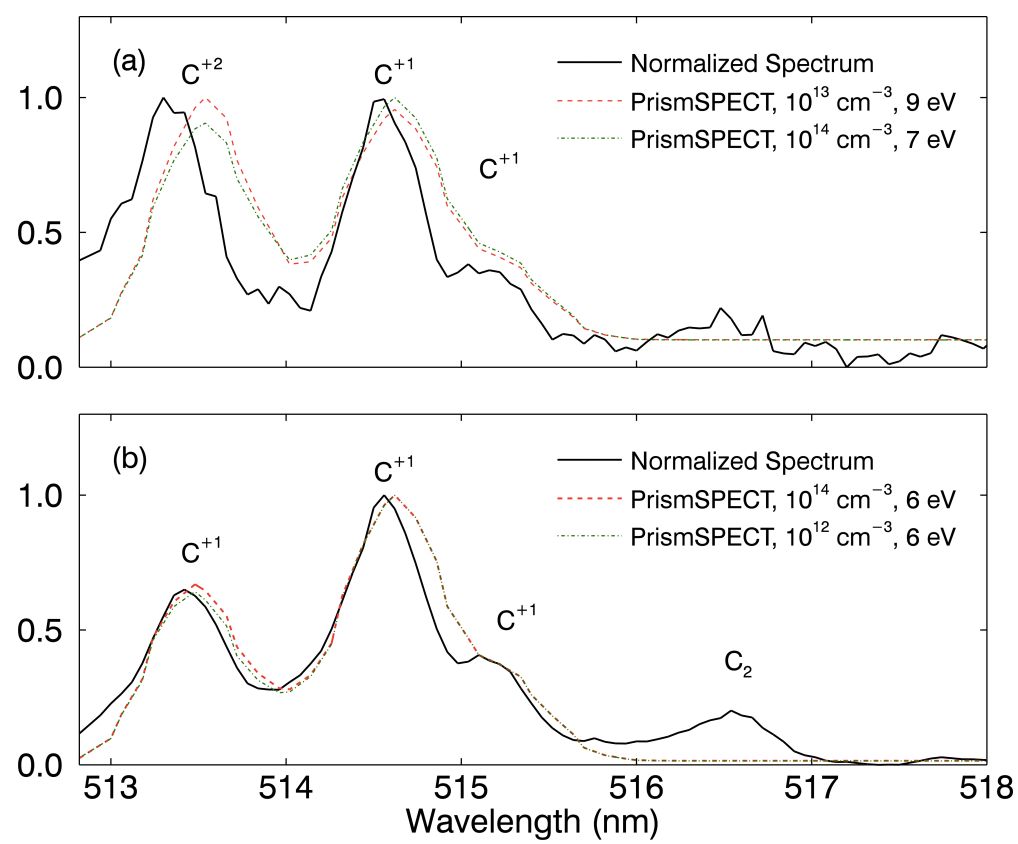

Figure 5: (Color Online) (a) Row-averaged profile (solid) of the carbon spectrum at $0.5 \mu$ s with PrismSPECT simulations (dashed). (b) Row-averaged profile (solid) of the carbon spectrum at 8.5 $\mu$ s with PrismSPECT simulations (dashed).

measurements from Thomson scattering and to estimate the ablated plasma's mean charge state. Since the densities were too low to satisfy local-thermodynamic-equilibirum (LTE) conditions, and hence calculate electron temperature and density from the Saha-Boltzman equation, the collisionalradiative code PrismSPECT [23] was used to simulate non-LTE spectra. Using PrismSPECT nonLTE, steady-state, instrument-broadened synthetic spectra for electron densities of $3 \times 10^{13}-3 \times$ $10^{14} \mathrm{~cm}^{-3}$ and electron temperatures of $7-9 \mathrm{eV}$ were found to be in good agreement with the spectrum at $0.5 \mu$ s (see Fig. 5a). Similarly, spectra with an electron temperature of $6 \mathrm{eV}$ over a large range of electron densities were found to be in good agreement with the spectrum at 8.5 $\mu$ s (see Fig. 5b). These values compare favorably to those calculated from Thomson scattering. The associated mean charge states calculated from PrismSPECT for the conditions from Thomson scattering were $\bar{Z}_{1}=3.9$ and $\bar{Z}_{2}=3.8$ for $0.5 \mu \mathrm{s}$ and $8.5 \mu \mathrm{s}$, respectively. This corresponds to ion densities of $1 \pm 0.5 \times 10^{14}$ at $0.5 \mu$ s and $5 \pm 3 \times 10^{13} \mathrm{~cm}^{-3}$ at $8.5 \mu \mathrm{s}$. The latter is consistent with an ion density of $3 \times 10^{13} \mathrm{~cm}^{-3}$ derived from the mass ablated by a heater intensity of $10^{11} \mathrm{~W} / \mathrm{cm}^{2}$ over $50 \mathrm{~ns}$ into a diamagnetic cavity of size $20 \mathrm{~cm}$ using the empirical mass ablation rate in [24].

The derived electron temperatures were also compared to laser-irradiated carbon simulations on HELIOS-CR, a 1-D radiation-magnetohydrodynamic code [25]. The simulations predicted electron temperatures of $\sim 10 \mathrm{eV}$, comparable to our calculated and spectral values, $3 \mathrm{~cm}$ from the target and $0.5 \mu \mathrm{s}$ after ablation. However, the simulations were significantly different $8.5 \mu \mathrm{s}$ after ablation, predicting an electron temperature of only $1 \mathrm{eV}$. This is most likely because HELIOS-CR could not account for a static background magnetic field. Generally, the relative stability in electron temperature seen over a $8 \mu$ s timescale differs significantly from trends observed elsewhere without a background magnetic field. In laser-ablated plasmas in vacuum, a simple model [26] 
and numerous experimental studies [26-28] have demonstrated that due to the expansion of the plasma, electron temperature scales with time $t$ as $T_{e} \propto t^{-2}$, or, if recombination dominates over expansion, $T_{e} \propto t^{-1}$. However, with a background magnetic field transverse to the blow-off direction, several new phenomena come into play, including plume confinement, plasma instabilities, and the conversion of plasma kinetic to thermal energy. Harilal et al. [29] have seen electron heating at the edge of a laser-driven plasma expanding into a magnetic field and have argued that electron cooling can be retarded due to resistive Ohmic heating and adiabatic compression by the magnetic field. Similar conditions appear to be in effect here, though over a larger spatial and temporal scale (see also [30]).

Finally, it is worth noting that at both $0.5 \mu \mathrm{s}$ and $8.5 \mu \mathrm{s}$, the Thomson spectrum has an overall redshift of $>0.55 \pm 0.27 \mathrm{~nm}$, with the error primarily from uncertainties in the etalon tuning of the probe beam frontend. This is opposite what one would expect with our scattering geometry, where a bulk plasma flow towards the collection optics would result in a blueshift (see Eq. 2.5), and currently remains unexplained.

\section{Summary}

We have applied Thomson scattering for the first time to the measurement of electron temperature and density in a large low-density, magnetized laser-plasma. The temperatures and densities were found to be relatively constant, even over the long experimental times, in contrast to trends observed without a magnetic field. Co-spectral carbon emission lines provide further evidence of this trend. This stability is attributed to the confinement provided by and currents generated by the magnetic field.

The large spatial and temporal scales probed here are of further interest for astrophysicallyrelevant, laboratory-simulated collisionless shocks, where large spatial (several ion gyro-radii) and temporal (several ion gyro-periods) scales are required to form and propagate the shock [31, 22]. This study presents the first step in measuring the temperature and density evolutions of these phenomena.

\section{Acknowledgments}

We would like to thank the staff of the Trident laser facility for their invaluable help. This work was supported by the LANL Trident laser facility and by the DOE/NSF Partnership in Basic Plasma Science under contract numbers DE-FG02-06ER5406 and NSF05-619. The work of SHG was performed under the auspices of the U.S. Department of Energy by Lawrence Livermore National Laboratory under Contract DE-AC52-07NA27344 and supported by LDRD grant 11-ER-050.

\section{References}

[1] N. Brenning, R. L. Merlino, D. Lundin, M. A. Raadu, and U. Helmersson, Faster-than-Bohm Cross-B electron transport in strongly pulsed plasmas, Physical Review Letters 103 (Nov., 2009) 225003.

[2] D. W. Koopman, High-beta effects and anomalous diffusion in plasmas expanding into magnetic fields, Physics of Fluids 19 (1976), no. 5670. 
[3] G. Dimonte and L. G. Wiley, Dynamics of exploding plasmas in a magnetic field, Physical Review Letters 67 (1991), no. 131755.

[4] A. Neogi and R. K. Thareja, Laser-produced carbon plasma expanding in vacuum, low pressure ambient gas and nonuniform magnetic field, Physics of Plasmas 6 (1999), no. 1 365-371.

[5] J. Sheffield, D. H. Froula, S. H. Glenzer, and N. Luhmann, Plasma Scattering of Electromagnetic Radiaiton. Academic Press, 2nd ed., 2011.

[6] S. H. Glenzer, W. E. Alley, K. G. Estabrook, J. S. D. Groot, M. G. Haines, J. H. Hammer, J. Jadaud, B. J. MacGowan, J. D. Moody, W. Rozmus, L. J. Suter, T. L. Weiland, and E. A. Williams, Thomson scattering from laser plasmas, Physics of Plasmas 6 (1999), no. 52117.

[7] D. E. Evans and J. Katzenstein, Laser light scattering in laboratory plasmas, Reports on Progress in Physics 32 (1969), no. 1 207-271.

[8] J. S. Ross, S. H. Glenzer, J. P. Palastro, B. B. Pollock, D. Price, G. R. Tynan, and D. H. Froula, Thomson-scattering measurements in the collective and noncollective regimes in laser produced plasmas (invited), Review of Scientific Instruments 81 (2010) 10D523.

[9] S. H. Glenzer and R. Redmer, X-ray thomson scattering in high energy density plasmas, Reviews of Modern Physics 81 (Dec., 2009) 1625.

[10] H. A. Baldis, Growth and saturation of the two-plasmon decay instability, Physics of Fluids 26 (1983), no. 51364.

[11] S. G. Belostotskiy, R. Khandelwal, Q. Wang, V. M. Donnelly, D. J. Economou, and N. Sadeghi, Measurement of electron temperature and density in an argon microdischarge by laser thomson scattering, Applied Physics Letters 92 (2008), no. 22221507.

[12] M. J. van de Sande and J. J. A. M. van der Mullen, Thomson scattering on a low-pressure, inductively-coupled gas discharge lamp, Journal of Physics D: Applied Physics 35 (2002), no. 12 1381-1391.

[13] D. E. Evans and P. G. Carolan, Measurement of magnetic field in a laboratory plasma by thomson scattering of laser light, Physical Review Letters 25 (Dec., 1970) 1605-1608.

[14] G. Jellison and C. R. Parsons, Resonant shadowgraph and schlieren studies of magnetized laser-produced plasmas, Physics of Fluids 24 (1981), no. 10 1787-1790.

[15] E. E. Salpeter, Plasma density fluctuations in a magnetic field, Physical Review 122 (June, 1961) 1663-1674.

[16] E. E. Salpeter, Electron density fluctuations in a plasma, Physical Review 120 (Dec., 1960) $1528-1535$.

[17] T. Lasspere, An Analysis of the Effect of an Imposed Magnetic Field on the Spectrum of Incoherent Scattering. PhD thesis, Cornell University, 1960.

[18] N. K. Moncur, R. P. Johnson, R. G. Watt, and R. B. Gibson, Trident: a versatile high-power nd:glass laser facility for inertial confinement fusion experiments, Appl. Opt. 34 (Jul, 1995) 4274-4283.

[19] C. Niemann, A. S. Bondarenko, C. G. Constantin, E. T. Everson, K. A. Flippo, S. A. Gaillard, R. P. Johnson, S. A. Letzring, D. S. Montgomery, L. A. Morton, D. B. Schaeffer, T. Shimada, and D. Winske, Collisionless shocks in a large magnetized laser-plasma plume, IEEE Transactions on Plasma Science 39 (Nov., 2011) 2406-2407. 
[20] E. T. Everson, P. Pribyl, C. G. Constantin, A. Zylstra, D. Schaeffer, N. L. Kugland, and C. Niemann, Design, construction, and calibration of a three-axis, high-frequency magnetic probe (B-dot probe) as a diagnostic for exploding plasmas, Review of Scientific Instruments 80 (2009), no. 11113505.

[21] S. Kacenjar, M. Hausman, M. Keskinen, A. W. Ali, J. Grun, C. K. Manka, E. A. McLean, and B. H. Ripin, Magnetic field compression and evolution in laser-produced plasma expansions, Physics of Fluids 29 (1986), no. 62007.

[22] Y. P. Zakharov, Collisionless laboratory astrophysics with lasers, IEEE Transactions on Plasma Science 31 (Dec., 2003) 1243-1251.

[23] J. MacFarlane, I. Golovkin, P. Wang, P. Woodruff, and N. Pereyra, Spect3d - a multi-dimensional collisional-radiative code for generating diagnostic signatures based on hydrodynamics and pic simulation output, High Energy Density Physics 3 (2007), no. 1-2 181 - 190.

[24] M. H. Key, W. T. Toner, T. J. Goldsack, J. D. Kilkenny, S. A. Veats, P. F. Cunningham, and C. L. S. Lewis, A study of ablation by laser irradiation of plane targets at wavelengths 1.05, 0.53, and 0.35 Iijm, Physics of Fluids 26 (1983), no. 72011.

[25] J. J. MacFarlane, I. E. Golovkin, and P. R. Woodruff, Helios-cr - a 1-d radiation-magnetohydrodynamics code with inline atomic kinetics modeling, Journal of Quantitative Spectroscopy and Radiative Transfer 99 (2006), no. 1-3 381-397.

[26] P. T. Rumsby and J. W. M. Paul, Temperature and density of an expanding laser produced plasma, Plasma Physics 16 (1974), no. 3247.

[27] B. C. Boland, F. E. Irons, and R. W. P. McWhirter, A spectroscopic study of the plasma generated by a laser from polyethylene, Journal of Physics B: Atomic and Molecular Physics 1 (Nov., 1968) 1180-1228-2.

[28] S. S. Harilal, C. V. Bindhu, R. C. Issac, V. P. N. Nampoori, and C. P. G. Vallabhan, Electron density and temperature measurements in a laser produced carbon plasma, Journal of Applied Physics $\mathbf{8 2}$ (1997), no. 5 2140-2146.

[29] S. S. Harilal, M. S. Tillack, B. O'Shay, C. V. Bindhu, and F. Najmabadi, Confinement and dynamics of laser-produced plasma expanding across a transverse magnetic field, Physical Review E 69 (Feb., 2004) 026413.

[30] A. B. Zylstra, C. Constantin, E. T. Everson, D. Schaeffer, N. L. Kugland, P. Pribyl, and C. Niemann, Ion velocity distribution measurements in a magnetized laser plasma expansion, Journal of Instrumentation 5 (June, 2010) P06004-P06004.

[31] C. Constantin, W. Gekelman, P. Pribyl, E. Everson, D. Schaeffer, N. Kugland, R. Presura, S. Neff, C. Plechaty, S. Vincena, A. Collette, S. Tripathi, M. Muniz, and C. Niemann, Collisionless interaction of an energetic laser produced plasma with a large magnetoplasma, Astrophysics and Space Science 322 (2009), no. 1 155-159. 